\title{
Autonomic Hyperreflexia after Spinal Cord Injury
}

\section{Freda C Richa*}

Saint-Joseph University, Hotel-Dieu de France Hospital, Anesthesia and Intensive Care Department, Beirut - Lebanon

\begin{abstract}
The most important complication of spinal cord lesions above T6 level is the phenomenon of Autonomic Hyperreflexia (AH). Symptoms and signs of $\mathrm{AH}$ result from the predominant parasympathetic excitation above the level of injury, and sympathetic excitation below the level of injury. Various noxious and nonnoxious stimuli below the level of injury can thus trigger off a mass autonomic response. The main triggering factor of $\mathrm{AH}$ is related with the urinary tract. The main treatment of $\mathrm{AH}$ is removal of the triggering factors. The development of intraoperative $\mathrm{AH}$ and hypertension can be prevented either by general anesthesia, which blunts autonomic reflexes, or regional anesthesia (spinal or epidural), which blocks afferent and autonomic efferent neural impulses.
\end{abstract}

Keywords: Autonomic hyperreflexia; Spinal cord injury

\section{Review}

The Autonomic Hyperreflexia $(\mathrm{AH})$ was first described in 1860 by Hilton [1] and the neuro-anatomical pathway was suggested by Kurnick in 1956 [2]. AH is developed in patients after severe spinal cord injury (SCI) above T6 level as a result of exaggerated spinal sympathetic excitation. It is caused by the spinal reflex mechanisms that remain intact despite the injury of the spinal cord $[3,4]$. The incidence of $\mathrm{AH}$ for spinal cord injuries above T6 is $85 \%$ [3-5].

\section{Physiopathology of AH}

$\mathrm{AH}$ is initiated by afferent impulses reaching the isolated spinal cord below the level of the spinal cord damage. While the nerve impulses travel up to the spinal cord, they are obstructed at the injury level. The input activates a reflex which increases the response of the sympathetic nervous system and gives a vasoconstriction and an hypertension [36]. The AH development has multifactorial complex mechanisms. Afferent impulses are carried by fibers which synapse within the dorsal grey matter of the spinal cord at various levels and ascend the dorsal and lateral columns until blocked at the level of SCI. As there is a loss of supraspinal control, the terminal boutons of presynaptic fibres divided by the cord transection become disorganized, leading to derangement in neighbouring, intact efferent fibres. Over the weeks following SCI, presynaptic boutons multiply, forming chaotic and inappropriate reflexes. Interneurones excited by the afferent inputs synapse with preganglionic sympathetic neurons in the intermediolateral grey column of the cord [4]. As a result of this process, an exaggerated reaction occurs within the preganglionic sympathetic neurones as a response to the afferent stimulus. The reason that $\mathrm{AH}$ is a feature of lesions at the T6 level or above is related with splanchnic circulation response to this sympathetic overactivity. The latter activity below the injury level results a splanchnic and peripheral vasoconstriction and causes hypertension. As a result of an excessive parasympathetic output above the level of the lesion, a peripheral vasodilation occurs $[5,7,8]$.

The brain is unable to influence the changes below the level of the injury, but above this level, the response of spinal centers is massive, leading to extensive sympathetic stimulation of the cardiovascular system and of the neurologically isolated adrenal medulla $[5,6]$. The denervated blood vessels are hypersensitive to any sympathetic stimulation and to the catecholamines released by the adrenal medulla [6]. This leads to vasoconstriction in the denervated area and compensatory vasodilation in the innervated area. Therefore, cord damage at T6 level or above is accompanied by increased secretion of adrenal medullary cathecholamines suggesting adrenaline implication in the development of the hyperreflexic responses as well as activation of adrenal sympathetic preganglionic neurons by visceral afferences leading to severe $\mathrm{AH}$. As a consequence of the extensive marked vasoconstriction, a hypertension occurs. A reflex bradycardia usually occurs through the intact vagus nerve and glossopharyngeal nerve (cranial nerves $\mathrm{X}$ and IX), because of the spinal cord transection does not interfere with afferent connections of the baroreceptors from the carotid sinus and aortic arch to the cardiac centers in the medulla oblongata $[3,5,6]$.

\section{Triggering}

Various noxious and nonnoxious stimuli below the level of injury can thus trigger off a massive autonomic response. Bors and French [9] reported that the greatest responses were produced by stimuli with the most caudal root levels below the region of SCI. This explains why pelvic visceral stimulation is the most commonly implicated $[1,7,10,11]$. Bladder distension is responsible for $75-82 \%$ of $\mathrm{AH}$ episodes [3]. Bladder distension may result from blocked or kinked indwelling urinary catheters (one of the commonest causes), urinary tract infection, interventional procedures and catheterization [7]. Other important causes are bowel distension, uterine contractions during obstetric delivery, acute abdominal pathology, fissure and urinary tract infection. Cutaneous and proprioceptive stimuli are less commonly implicated but manipulation of pressure sores, ingrown toenails and even sunburn have been known to trigger the phenomenon. During surgery under general anesthesia, various tiggers can precipitate $\mathrm{AH}$, such as tracheal intubation or extubation, pain and other surgical stimuli $[4,5,7]$.

\section{Symptoms and Signs of $\mathrm{AH}$}

Essentially symptoms and signs of AH result from the predominant parasympathetic excitation above the level of injury, and sympathetic excitation below the level of injury [3,5]. Usually, patients with SCI at T6 or above have normal systolic blood pressure of $90-110 \mathrm{mmHg}$. A sudden 20 to $40 \mathrm{mmHg}$ increase of systolic and diastolic blood pressure over baseline that is frequently associated with bradycardia

*Corresponding author: Freda Richa, Hotel-Dieu de France Hospital, Anesthesia and Intensive Care Department, Alfred Naccache street, Ashrafieh, Beirut, 0020 Lebanon, Tel: 009613872077; Fax: 009611615295; E-mail: fredrich24@yahoo.com

Received November 07, 2014; Accepted December 17, 2014; Published December 19, 2014

Citation: Richa FC (2014) Autonomic Hyperreflexia after Spinal Cord Injury. J Spine 4: 196. doi:10.4172/2165-7939.1000196

Copyright: $@ 2014$ Richa FC. This is an open-access article distributed under the terms of the Creative Commons Attribution License, which permits unrestricted use, distribution, and reproduction in any medium, provided the original author and source are credited. 
may indicate AH. A noxious stimulus below the level of the lesion produces an afferent impulse that generates a generalized sympathetic response, which in turn results in widespread vasoconstriction, most significantly in the splanchnic vasculature, which causes an increase in peripheral resistance and a shunting of the normal blood that is congested, thereby forcing it to enter into the general circulation. The combination of the increased vasoconstriction and the increased fluid load in the vascular space causes a potentially catastrophic increase in blood pressure (BP). Systolic BP can increase to as high as $300 \mathrm{~mm} \mathrm{Hg}$, diastolic BP to as high as $200 \mathrm{~mm} \mathrm{Hg}$. The brain detects this hypertension crisis through intact baroreceptors and stimulates the parasympathetic nervous system in an attempt to lower BP. The parasympathetic overactivity (and lack of sympathetic tone) above the level of the lesion results in peripheral vasodilation and is thought to be responsible for the headache, flushing and sweating in the head and neck region, and the nasal congestion $[7,12]$. Other associated symptoms are nausea, anxiety, malaise, Horner's syndrome (partial ptosis, miosis, anisocoria, hemifacial anhidrosis) and penile erections. Signs on examination include sweating, tremor, blushing and piloerection above the level of the lesion. Twitching and increased spasticity in all limbs may be shown [5]. The cardiovascular responses associated with $\mathrm{AH}$ are complex and variable. Tachycardia or reflex bradycardia are most commonly observed in this setting, and are attributed to heightened noradrenergic sympathetic activity as well as reflex vagal responses. Other electrocardiographic abnormalities have been observed during an attack of $\mathrm{AH}$ such as premature atrial and ventricular contractions, T-wave and $\mathrm{U}$-wave amplitudes alterations, atrial fibrillation, bigeminy, prolonged P-R interval and conduction block $[13,14]$. Moreover, the arrhythmias associated with AH have seldom been recognized as a cause of cardiac arrest in individuals with SCI. Cholachis et al. report a rare description of AH-inducing recurrent ventricular fibrillation in a patient with C6 tetraplegia [13]. The hypertension in $\mathrm{AH}$ is paroxysmal and may be severe leading to subarachnoid, intracranial and retinal haemorrhages, seizures, coma, myocardial ischemia, pulmonary oedema and death [4,5,13-16].

\section{Treatment}

\section{Nonpharmacologic management of AH}

The first step of treatment, regardless of the cause, is to place the patient in an upright position, thus pooling the blood in the abdominal and lower extremity vessels, due to the loss of peripheral vasoconstriction that follows SCI, thereby causing a reduction in arterial blood pressure. Thereafter, the precipitating stimulus should be searched and removed thus blood pressure often returns to baseline levels immediately [13]. In particular, the urinary tract irritation or distention and fecal impaction are the most common triggers of $\mathrm{AH}$, must be checked urgently and regularly [3,11]. Various methods have been tried to control $\mathrm{AH}$ by interrupting the reflex arc at different levels extending from the origin of the noxious stimuli to the cardiovascular system target site [6]. A local anesthetic jelly is used to unblock an indwelling catheter in situ, and then the drainage bag and the catheter should be inspected. The bladder should be palpated in case of distended patient using intermittent catheterization. Moreover, the rectum should be examined and emptied by gentle insertion of a gloved finger, lubricated in lidocaine jelly. A lidocaine jelly- lubricated suppository or enema tip can also be used to evacuate the rectum. Sometimes, a full enema may be needed. After ruling out the bladder and bowels problems, skin ulceration or a musculoskeletal disturbance may be alternative triggers [7].

\section{Pharmacologic management of AH}

If the non-pharmacological measures fail, several pharmacologic agents, including intravenous ganglion blockers, hydralazine, $a$-adrenergic receptor blockers, calcium channel blockers, or nitrates, have been used in the prevention or control of $\mathrm{AH}$, although these agents are not always safe, convenient, or predictable. Antihypertensive medication should preferably have a rapid onset and short duration of action. Nifedipine or nitrates are the most commonly used medications during acute attack [4,5,17-19].

\section{General Anesthesia}

The development of intraoperative $\mathrm{AH}$ and hypertension may be prevented either by general anesthesia, which blunts autonomic reflexes, or regional anesthesia, which blocks afferent and autonomic efferent neural impulses. Patients with SCI often have a lower blood volume and a reduced lean tissue mass as a result of muscle wasting. This implies a smaller volume of distribution for intravenous anesthetic agents and a smaller vessel-rich compartment. This explains the greater observed sensitivity of these patients to intravenous induction agents. The effect is compounded by an absence of reflex sympathetic activity, reducing the ability to compensate for myocardial depressant effects. SCI is often associated with renal impairment, which may result in reduced clearance of some drugs [5]. Many drugs commonly used during anesthesia are dependent to some degree on renal excretion for elimination, and this must be taken into consideration when planning an anesthetic for patients with renal dysfunction. These patients are sensitive to barbiturates and benzodiazepines secondary to decreased protein binding. Some narcotic agents including morphine and meperidine should be used carefully as they have active metabolites and may have prolonged activity in the setting of renal dysfunction. Fentanyl and hydromorphone are better choices. Cisatracurium and atracurium are nondepolarizing muscle relaxants that do not rely on renal function for their elimination, but are metabolized by ester hydrolysis and Hoffmann elimination. Neuromuscular reversal agents rely on renal excretion and, therefore, their effects will be prolonged. Many antimicrobial agents must be dosed according to renal function. Nonsteroidal antiinflammatory agents should be avoided [20]. All induction agents are useful except for the Ketamine which carries the theoretical risk of worsening muscle spasm [5].

Concerning the suxamethonium, since the appearance of many papers in the early 1970s, there have been very few studies on its use in SCI. A particular area of controversy is the duration of denervation hypersensitivity and when the use of suxamethonium may safely resume after injury. Reported cases of cardiac arrest were attributed to hyperkalemia induced by suxamethonium within the 6 months period of the injury. During this period, the use of this drug could possibly be justified just for a specified tough indication such as a rapid sequence induction with difficult intubation $[4,7]$.

During general anesthesia, the use of muscle relaxants is necessary firstly to facilitate the tracheal intubation and secondly to optimize the surgical field by causing relaxation of skeletal muscles. The use of muscle relaxants is not necessary if the patient present a severe muscular wasting. As spinal lesion induced neuromuscular changes, monitoring of neuromuscular blockade (by evoked muscle response to nerve stimulation) is recommended when their use is needed to avoid residual paralyses [5].

Saito et al. [21] described a 26-year-old male patient, who developed $\mathrm{AH}$ while undergoing colostomy under general anesthesia. These authors recommended nicardipine for treatment of $\mathrm{AH}$ in a 
patient with upper SCI undergoing surgery under general anesthesia. Sevoflurane has been used to block AH during transurethral litholapaxy under general anesthesia in patients with complete SCI. Yoo et al. [10] studied 28 patients with chronic, complete SCI, who were scheduled to undergo transurethral litholapaxy during general anesthesia. These researchers found that the end-tidal concentrations of Sevoflurane to prevent $\mathrm{AH}$ in patients were EC50 of 3.12\% and EC95 of 3.83\% (the effective concentrations to block systolic blood pressure response in $50 \%$ and $95 \%$ of patients). Their findings indicate that a deep level of anesthesia is required to block AH. A deep general anesthesia with systemic opioids is recommended to prevent autonomic responses in the face of noxious stimulation and to blunt the sympathetic response during surgery $[10,11]$.

\section{Regional Anesthesia}

Subarachnoid and epidural anesthesia have been successfully used to prevent the triggering of $\mathrm{AH}$ by blocking the afferent nerves, the spinal neurons, and the efferent nerves $[5,6,22]$. Therefore, epidural anesthesia is preferable because in spinal anesthesia the onset of sympathetic blockade is rapid and block height cannot always be predicted. Patients with chronic spinal injuries have low resting blood pressure due to impaired autoregulatory function. In high lesions, the ability to prevent orthostatic falls in blood pressure and to increase heart rate via the cardioaccelerator fibres $(\mathrm{T} 1-4)$ is lost $[5,6,22]$. The most widely accepted technique to control $\mathrm{AH}$ is epidural analgesia, although the choice of a certain drug, its concentration, and its volume is controversial. An epidural narcotic seem to be a good choice because it will interrupt the reflex arc by blocking the nociceptive impulses while sparing sympathetic tone that can be quite compromised by the cord damage $[6,22]$.

Epidural anesthesia for labor and delivery in a patient with $\mathrm{AH}$ was first described in 1979 by Stirt et al. [23]. The technique allows for a slower rise in the sympathetic blockade, while block height can be controlled by small incremental doses of local anesthetic. Epidural pethidine alone has been successfully used to control $\mathrm{AH}$ in spontaneous labor [24]. The use of fentanyl alone during epidural has been unsuccessful due to the lack of local anesthetic action [4]. The combination of bupivacaine and fentanyl was described by Gunaydin et al. [25] for caesarean section in patients with SCI.

The test dose for accidental subarachnoid placement is difficult to interpret given the existing extensive motor and sensory block. The ability to elicit muscle spasms in response to ethyl chloride spray has been described as a method of judging block level [4]. The use of epinephrine-containing solutions in testing for accidental intravascular placement has been suggested, but it was avoided because of the uncontrolled hypertension risk [20].

In conclusion, for all operations on cord-injured patients, it is recommended that the usual precautions should be taken, such as the presence of the anesthetist, a secured venous access, a continuous monitoring throughout the procedure. An adequate anesthetic technique (general or regional) should be considered to blunt the sympathetic response during surgery, avoiding then the occurrence of $\mathrm{AH}$.

\section{References}

1. Hilton $J(1860)$ A course of lectures on pain and therapeutic influence of mechanical and physiological rest in accidents and surgical diseases. Lancet 2: $401-404$.

2. Kurnick NB (1956) Autonomic hyperreflexia and its control in patients with spinal cord lesions. Ann Intern Med 44: 678-686.

3. Vaidyanathan S, Soni B, Selmi F, Singh G, Esanu C, et al. (2012) Are urological procedures in tetraplegic patients safely performed without anesthesia? A report of three cases. Patient Safety in Surgery 6: 3.
4. Hambly PR, Martin B (1998) Anaesthesia for chronic spinal cord lesions. Anaesthesia 53: 273-289.

5. Petit JS, Delahaye JM, Malinovsky JM (2008) [Perioperative management of traumatic spinal cord injured patients after the acute phase]. Ann Fr Anesth Reanim 27: 416-425.

6. Abouleish El, Hanley ES, Palmer SM (1989) Can epidural fentanyl control autonomic hyperreflexia in a quadriplegic parturient? Anesth Analg 68: 523526 .

7. Bycroft J, Shergill IS, Chung EA, Arya N, Shah PJ (2005) Autonomic dysreflexia: a medical emergency. Postgrad Med J 81: 232-235.

8. Weaver LC, Marsh DR, Gris D, Brown A, Dekaban GA (2006) Autonomic dysreflexia after spinal cord injury: central mechanisms and strategies for prevention. Prog Brain Res 152: 245-263.

9. Bors E, French JD (1952) Management of paroxysmal hypertension following injuries to cervical and upper thoracic segments of the spinal cord. AMA Arch Surg 64: 803-812.

10. Yoo KY, Jeong CW, Kim SJ, Chung ST, Bae HB, et al. (2008) Sevoflurane concentrations required to block autonomic hyperreflexia during transurethra litholapaxy in patients with complete spinal cord injury. Anesthesiology 108 : 858-863.

11. Yoo KY, Jeong CW, Kim SJ, Jeong ST, Kim WM, et al. (2011) Remifentanil Decreases Sevoflurane Requirements to Block Autonomic Hyperreflexia During Transurethral Litholapaxy in Patients with High Complete Spinal Cord Injury. Anesth Analg 112: 191-7.

12. Gunduz H, Binak DF (2012) Autonomic dysreflexia: an important cardiovascular complication in spinal cord injury patients. Cardiol J 19: 215-219.

13. Colachis SC 3rd, Clinchot DM (1997) Autonomic hyperreflexia associated with recurrent cardiac arrest: case report. Spinal Cord 35: 256-257.

14. Jain A, Ghai B, Jain K, Makkar JK, Mangal K, et al. (2013) Severe autonomic dysreflexia induced cardiac arrest under isoflurane anesthesia in a patient with lower thoracic spine injury. J Anaesthesiol Clin Pharmacol 29: 241-3.

15. Eker A, Yigitoglu PH, Ipekdal HI, Tosun A (2014) Acute Onset of Intracerebral Hemorrhage due to Autonomic Dysreflexia. J Korean Neurosurg Soc 55: 277-279.

16. Yoo KY, Jeong CW, Kim WM, Lee HK, Kim SJ, et al. (2010) Fatal cerebra haemorrhage associated with autonomic hyperreflexia during surgery in the prone position in a quadriplegic patient: a case report. Minerva Anestesiol 76: $554-8$

17. Schonwald G, Fish KJ, Perkash I (1981) Cardiovascular complications during anesthesia in chronic spinal cord injured patients. Anesthesiology 55: 550-558.

18. Stowe DF, Bernstein JS, Madsen KE, McDonald DJ, Ebert TJ (1989) Autonomic hyperreflexia in spinal cord injured patients during extracorporeal shock wave lithotripsy. Anesth Analg 68: 788-791.

19. Lambert DH, Deane RS, Mazuzan JE Jr (1982) Anesthesia and the control of blood pressure in patients with spinal cord injury. Anesth Analg 61: 344-348.

20. Wagener G, Brentjens TE (2010) Anesthetic concerns in patients presenting with renal failure. Anesthesiol Clin 28: 39-54.

21. Saito J, Kimura F, Ishihara H, Hirota K (2009) [Case of autonomic hyperreflexia treated with intravenous nicardipine]. Masui 58: 1528-1530.

22. Burns R, Clark VA (2004) Epidural anaesthesia for caesarean section in a patient with quadriplegia and autonomic hyperreflexia. Int J Obstet Anesth 13: $120-123$.

23. Stirt JA, Marco A, Conklin KA (1979) Obstetric anesthesia for a quadriplegic patient with autonomic hyperreflexia. Anesthesiology 51: 560-562.

24. Baraka A (1985) Epidural meperidine for control of autonomic hyperreflexia in a paraplegic parturient. Anesthesiology 62: 688-690.

25. Gunaydin B, Akcali D, Alkan M (2001) Epidural anaesthesia for Caesarean section in a patient with Devic's Syndrome. Anaesthesia 56: 565-567. 